\title{
Interoperability of Messenger Services. Possibilities for a Consumer-Friendly Approach
}

Jörg Becker, Bernd Holznagel, Kilian Müller ${ }^{1}$

Abstract: Messenger services connect consumers around the globe on a daily basis and help them share news, photos, videos and other information. To communicate, however, users need a shared messenger service. Information exchange between different service providers is rarely possible. Due to network effects, this leads to an increasing monopolisation of the messenger service market. Users are increasingly forced to use messenger services from a single manufacturer, which further extends its supremacy. Therefore, it is investigated to what extent the introduction of an interoperability obligation could counteract these effects in a consumer-friendly way. As a possible solution, the introduction of a federal XMPP-based system is presented, which could be used to implement an interoperability obligation in practice.

Keywords: Platform Regulation, Messenger Services, Federated Protocols, Competition, Interoperability and its legal possibilities under EU law

\section{Chapter 1: Introduction}

Messenger services are becoming increasingly popular. While in 2016, around $67 \%$ of all users aged 14 and above used messenger services ${ }^{2}$, this figure increased to almost $90 \%$ in 2018 . The figure is even higher among younger people aged between 14 and 29, where almost everyone (98\%) now uses messenger services. ${ }^{3}$ Facebook holds the largest market share. WhatsApp alone accounts for $96 \%$ of usage share. The second most-

1 The study was funded by the German Federal Ministry of Justice and Consumer Protection based on a resolution of the German Bundestag.

2 "Zwei von drei Internetnutzern verwenden Messenger", bitkom, accessed June 23, 2021, https:/www.bitkom.org/Presse/Presseinformation/Zwei-von-drei-Internetnut zern-verwenden-Messenger.html.

3 „Zwei von drei Internetnutzern“. 
used communication service, also from Facebook, is Facebook Messenger with $42 \%{ }^{4}$ This market position enables Facebook to exercise a dominant position on the market, which is increasingly strengthened by existing network effects. These network effects increase the benefit of a particular service for all users involved as the number of users increases. In other words, the more users already use a service, the more attractive it becomes for new users, and for each new user the benefit of all existing users increases. In extreme cases, this can lead to so-called lock-in effects, through which consumers cannot move to another service provider without inconvenience.

One way to prevent or disrupt such a monopolistic position is to impose an interoperability obligation. If an interoperability obligation were to apply in the messenger service market, as introduced by the EECC (see chapter $\mathrm{C}$ ), users would have to be able to exchange messages with users of other services without having to install the respective service. Thus, they can use their chosen service to contact users of all other services. Therefore, it will be investigated how an interoperability obligation can be technically executed and how it affects competition, innovativeness, data privacy, and usability for consumers.

In the following, the subjects of discussion (B I.), as well as the functionalities and the interoperability of messenger services, are presented (B II.). This is followed by an evaluation of the interoperability concept (B III.). In B IV, the design options for a successful introduction of interoperability are explained in more detail. Subsequently, possible interoperability obligations under the EKEK, the TKMoG-E, and the GWG-E are addressed (C I-III.). The paper ends with a conclusion (D).

4 Bundesnetzagentur für Elektrizität, Gas, Telekommunikation, Post und Eisenbahnen, Nutzung von OTT-Kommunikationsdiensten in Deutschland (Bonn: Bundesnetzagentur für Elektrizität, Gas, Telekommunikation, Post und Eisenbahnen, 2020), https://www.bundesnetzagentur.de/SharedDocs/Mediathek/Berichte/2020/OTT.pdf ?_blob=publicationFile. 


\section{Chapter 2. Technical/economical view}

\subsection{Subject}

\subsubsection{Messenger services}

The German Federal Network Agency ("Bundesnetzagentur") classifies messenger services and other digital platforms in the category of "over-thetop" (OTT) services, which enable communication and other services via the Internet. ${ }^{5}$ This means that, in contrast to traditional telephony and text-messaging services, the use of OTT services is not tied to the respective mobile or landline connection. They, therefore, do not require their own infrastructures but use existing infrastructures to build their services on. These services include for example sending messages, displaying a status, or sending images or video material.

The key task of messenger services is to connect users for the exchange of messages and other information. In contrast to social networks such as Facebook, YouTube, or Instagram, in which content can be broadcast by a sender and then be displayed to various consumers, in messenger services the addressee is specifically selected, which means that messages can only be received by this person. The message is sent via various open communication protocols such as XMPP, IRC, or Echo, or via proprietary protocols such as WhatsApp or Skype over the public Internet using a client. An addressee who has the same client or the same protocol can receive and read this message. Open protocols thus open up the theoretical possibility of connecting users from different clients with each other, whereas proprietary protocols usually exclude this possibility and only allow communications between users of their own client.

\subsubsection{Interoperability}

Communication between users of two different clients would constitute interoperable communication. In general terms, interoperability refers to the ability of different systems to communicate with each other and to be able to use the communicated information. ${ }^{6}$ In a technical context,

5 Bundesnetzagentur, Nutzung von OTT-Kommunikationsdiensten.

6 "Stellungnahme der Digitalen Gesellschaft e. V. zur Konsultation des Bundesministeriums der Justiz und für Verbraucherschutz zu Interoperabilität und Daten- 
interoperability includes the ability of two or more software components to work together despite differences in language, interface, and execution environment. In the context of messenger services, interoperability refers to the possibility of exchanging messages and other types of communication not only between users of the same messenger A, but also between different messenger services A and B. According to Wegner, the two main mechanisms for creating interoperability are the creation of interfaces and standardization, with standardization being more scalable and interfaces being more flexible. ${ }^{7}$ The use of a common communication protocol would also mean standardization of functionalities. The use of interfaces would make certain functionalities interoperable, independent of protocol. In this case, it could be individually selected which functionalities and data are passed on.

\subsection{Introducing Interoperability}

As stated above, according to Wegner, there are basically two ways to ensure interoperability between messenger services, standardization and the creation of interfaces. ${ }^{8}$ The creation of federated systems uses an interface to mediate between different protocols or domains, using a common standard. A federation can be seen as a combination of both approaches.

\subsubsection{Interfaces}

One way to enable interoperability between messenger services is to create interfaces called application programming interfaces (APIs). APIs are data processing interfaces that define the interactions between multiple software components. APIs provide only the data that the other software component needs or requests. Using standardized formats, such as JSON or XML, certain components, such as the text content of a message, its re-

portabilität bei sozialen Netzwerken", Digitale Gesellschaft e. V., accessed June 23, 2021, https://digitalegesellschaft.de/2019/05/stellungnahme-der-digitalen-gesellscha $\mathrm{ft}$-e-v-zur-konsultation-des-bundesministeriums-der-justiz-und-fuer-verbrauchersch utz-zu-interoperabilitaet-und-datenportabilitaet-bei-sozialen-netzwerken/.

7 Peter Wegner, "Interoperability", ACM Computing Surveys 28, no. 1 (March 1996): 285 ff., https://doi.org/10.1145/234313.234424.

8 Wegner, "Interoperability", $285 \mathrm{ff}$. 
cipient, or telephone numbers, could be exchanged automatically between two or more different service providers.

\subsubsection{Standardization}

Standardization of communication protocols is another option for enabling interoperability between messenger services. Many functionalities of the messenger services depend on the respectively used protocols. It must therefore be ensured that a protocol is selected or created that supports all the functionalities that are to be interoperable. For this purpose, each messenger service must change its implementations to the new protocol or support multiple protocols.

\subsubsection{Federation}

In the case of a federation, the individual messenger services could retain their own protocols. However, a standardized protocol is used to which different parts of the respective messenger protocols can be "mapped". Thus, messages sent by users with the same messenger can still be transmitted using the messenger's own protocol. However, if a message is sent that is addressed to a user with a different messenger service, the interoperable parts of this message are passed to the federated protocol, transmitted to the target messenger, and finally forwarded to the recipient. For this purpose, a unified encryption protocol must be used to ensure end-to-end encryption.

\subsection{Impact of interoperability}

As interfaces are becoming increasingly impractical with an increasing number of participants and standardization hinders innovativeness the introduction of a federated system is examined with regard to its impact on competition, innovativeness, data privacy, and usability has to be examined. 


\subsubsection{Competition}

Messenger services, being digital platforms, benefit from network effects. The more users such a digital platform has, the greater its benefit for all users involved (direct network effects). ${ }^{9}$ This can influence new users in particular, as they increasingly opt for the largest provider in order to gain the greatest benefit from existing network effects. Furthermore, digital platforms with a large user base can use their existing network to expand into other areas by using the existing database for other purposes. This allows a company to continuously improve a product through the existing network (economies of scope), an opportunity not available to competitors with smaller networks. Another advantage for Facebook results from the use of indirect network effects. For both advertising and analysis purposes, Facebook can draw on a significantly larger user and database and thus generate further advantages over smaller competitors. In general, it should therefore be the case that the more users a messenger service has, the more useful this service is for all parties involved. ${ }^{10}$ However, indirect network effects can put the users of a service at a disadvantage due to increased advertising or other use of their personal data. This is particularly problematic if the market share of a single service is sufficiently large, as this can lead to lock-in effects that make the user dependent on the respective service if there is no sufficient other alternative. As already mentioned, Facebook has market shares of over $90 \%$. If "Facebook-external" users, i.e., users without a messenger service from Facebook, want to communicate with other users who use a Facebook messenger service such as WhatsApp, they are currently forced to use a messenger service from Facebook. Installing another shared messenger service on both sides (multihoming) would be an alternative, though it would involve additional effort. If consumers do not use a Facebook messenger service, they will not be able to reach most of their contacts via another messenger service unless the other person has installed another messenger service. In $65 \%$ of the cases, users have at least two different messenger services installed. ${ }^{11}$ However, this does not always have to be the same additional messenger service, which would force consumers to install more than two different messenger services. The installation of multiple messenger services, though, consumes more

9 Michael L. Katz and Carl Shapiro, "Systems Competitionand Network Effects", Journal of Economic Perspectives 8, no. 2 (Spring 1994): 93 ff., https://doi.org/10.125 7/jep.8.2.93.

10 Katz and Shapiro, "Systems competition", $93 \mathrm{ff}$.

11 Bundesnetzagentur, Nutzung von OTT-Kommunikationsdiensten. 
storage space, causes a wider distribution of personal data, and ultimately forces users to install a Facebook messenger service anyway in order to achieve full availability. At this point, it should be emphasized that multihoming can of course be used to achieve almost full availability, but this is never possible without a Facebook Messenger service. Users are thus faced with the decision of passing on their personal data to Facebook or giving up full availability. To strengthen broader competition independent of Facebook, it is necessary to break up the prevailing network effects. Generally speaking, any form of interoperability obligation supports the intention to mitigate network effects. By enabling users to communicate with users of other services, they are no longer dependent on the provider with the largest user base but can select the messenger service according to other criteria. Functionality, graphical user interface, etc. are already criteria by which users select a messenger service, but they currently play a minor role compared to network effects. ${ }^{12}$ An introduction of an interoperability obligation could therefore strengthen competition based on new functionalities or better interfaces. Furthermore, this would give new companies a better chance to enter the competition, as they are not measured by the size of their user base, but by their functionalities, data privacy, etc. However, an interoperability obligation must protect the vendors' Unique Selling Points (USPs) to ensure a justification for smaller vendors to exist. In terms of competition, no differences are expected regarding the three different design options.

\subsubsection{Innovativeness}

Innovativeness in the context of messenger services refers to the ability to continuously create new functionalities or to continuously improve existing functionalities. One incentive for companies to be innovative is the resulting competitive advantages. However, these would be lost if other competitors were able to adapt or copy an innovation immediately without major difficulties. New functionalities, for example, require considerable implementation and testing effort before they can be introduced in a stable form. It should also be noted that too much market power could cause new/small companies to shy away from investing in innovations, as these are unlikely to pay off.

12 Katz and Shapiro, "Systems competition", $93 \mathrm{ff}$. 
A federation circumvents the disadvantages of standardization in terms of innovativeness by still allowing companies to use their own protocols for intra-messenger communication. Only inter-messenger communication relies on a standardized protocol, to which functionalities can of course be added. However, messenger services can implement their own innovations in their own protocols and use these only for intra-messenger communications, thus maintaining their competitive advantage and continuing to generate incentives for future innovations. This avoids the problem that new functionalities based on a completely standardized protocol take a long time to be implemented. There would only be an additional effort in terms of implementation if further additional functionalities were made interoperable in the future, as these would then also have to be provided in inter-messenger communication.

\subsubsection{Data privacy}

Regarding data privacy, a distinction can be made between several aspects. On the one hand, end-to-end encryption can suffer from an interoperability obligation if no common encryption protocol is used, and the message thus must be decrypted and re-encrypted in several steps. However, this can be circumvented by using a common encryption protocol. Since intermessenger messages automatically involve two companies in the communication, they inevitably generate more metadata than intra-messenger communications. The sending service needs information about the recipient of the message to deliver it. When the addressee replies, the service responsible for this also requires information about the original sender. The generated personal data is, however, significantly less (fewer providers are required) than when using multihoming, since the messenger service that is not installed and involved in the communication only obtains information about the username of the unknown user, but ideally does not obtain any further information due to end-to-end encryption. Thus, information about the address book, access to photos, etc. could be kept secret from the other messenger service. The transmission of the data by the sender and the storage of the data by the recipient qualify as processing within the meaning of Art. 4 No. 2 GDPR. Both must comply with the requirements of Art. 6(1) GDPR. Furthermore, the recipient is subject to the information obligations resulting from Art. 14 GDPR. In addition, the principles relating to processing of personal data according to Art. 5 GDPR must be considered when establishing interoperability. Of importance in this context are the principles of good faith, data minimization, data security, and 
transparency. They must be considered when designing the interoperability system. Which remains true for federated systems.

WhatsApp already uses FunXMPP, an XMPP-based protocol that enables federated communication. To send a message to a user of another messenger service, the sender must know the exact address of the addressee. Similar to an email address, this is composed of a username and a domain (Username@Domain.de). WhatsApp, for example, replaces the username with the respective cell phone number. All common XMPP servers provide functionalities that support end-to-end encryption of messages between multiple clients, e.g., with OMEMO or other extensions. Using end-to-end encryption, the operators of the messenger services only know the addressee, message type, and time of message transmission, but the content of the messages remains hidden.

\subsubsection{Usability}

Each messenger service can implement functionalities that other messenger services do not support. If these functionalities are partially interoperable, consumers may miss familiar functionalities. For example, if a messenger service of an addressee does not support video telephony, or if video telephony, in general, should not be part of the interoperability regulation, consumers might be confused why this normally familiar functionality is not available to them. Furthermore, sharing data with yet another provider could discourage users from participating in inter-messenger communications. Different messenger services embody different types of emotional proximity of the communicators. ${ }^{13}$ Arnold et al ${ }^{14}$ note that users distinguish which messenger service or type of communication they used to communicate with certain people. However, this is only possible if the respective addressees have the selected messenger service or type of communication. An interoperability obligation would eliminate the choice of messenger service depending on the addressee and emotional proximity and could thus limit the user experience. It is questionable at this point, though, whether this is to be understood as a user experience or

13 René Arnold and Anna Schneider, "An App for Every Step: A psychological perspective on interoperability of Mobile Messenger Apps", 28th European Regional Conference of the International Telecommunications Society (ITS) (July/August 2017).

14 René Arnold et al., "Interoperability of interpersonal communications services A consumer perspective", Telecommunications Policy 44, no. 3 (April 2020), https:// doi.org/10.1016/j.telpol.2020.101927. 
a necessity. The argument is supported by a report of the Federal Network Agency. ${ }^{15}$ Here, $53 \%$ of the users surveyed stated that they did not see any need to be able to contact users of other messenger services directly. However, $45 \%$ of the respondents would like to have this functionality. $67 \%$ of the respondents, though, would not like to be contacted directly by users of other messenger services. This would mainly be due to the parallel use of different free messenger services, which do not make interoperability necessary. This hypothesis should be questioned, though, since users might find it difficult to imagine interoperability of messenger services and thus no accurate statement can be made regarding actual future use.

The ability to communicate with users outside the same messenger service without having to install a new service, therefore, does not yet seem to be explicitly desired by consumers. This can also be attributed to a lack of experience in this area, though, and would have to be analysed in further scientific studies in case of an interoperability obligation.

\subsection{Result}

The introduction of an interoperability obligation would break up any existing network effects. This would particularly benefit small or new companies, as it would make it easier for them to enter the market. However, other competitive advantages or USPs should be protected to ensure continued innovativeness.

Standardization restricts the innovativeness and thus also the resulting competition too much. Creating interfaces for all other services requires an increased administrative effort for each newly added messenger service. What all these federations have in common is that the number of connections to be realized between the systems involved is $2 * \mathrm{~N}(\mathrm{~N}$ is the number of systems involved), i.e., it grows linearly with the number of systems. For interfaces, the number of connections to be realized is $\mathrm{N}^{*}(\mathrm{~N}-1)$, so it grows polynomially with the number of systems. This also speaks for federations instead of interfaces. Therefore, a federated system avoids these disadvantages and creates a platform on which companies can on the one hand maintain their own strengths (their own extended protocols for intra-messenger communication) and on the other hand are open to other providers to mitigate network effects. 
As described above, an XMPP-based, federated system offers the possibility of establishing interoperability between different messenger services. XMPP is open source, so it does not belong to any company, and it can be extended by further services or functionalities. This means that additional functionalities that are not part of the general XMPP standard can be added and used within a messenger (see FunXMPP). Interoperable functionalities must be incorporated into the standard XMPP protocol in order to be generally accessible and thus interoperable. Via gateways, XMPP partially allows communication with non-XMPP-based messenger services, so-called legacy services. ${ }^{16}$ These gateways could also be used to exchange messages between domains with more advanced XMPP-based protocols, such as WhatsApp, and domains using the standard protocol. However, the creation of these gateways requires a noticeable implementation effort, which must be considered in the context of the proportionality assessment of an order of the interoperability obligation by the Federal Network Agency.

Users of a messenger service would only need to know the XMPP address of their contact to be able to contact him across domains. Existing apps such as Quicksy ${ }^{17}$ or Zom ${ }^{18}$ can already contact services of other providers outside their own domains that use XMPP. In order to be able to contact WhatsApp users as well, another gateway solution is required.

Federations have already been used successfully in other areas and thus can help messenger services to become interoperable. Examples include the conversion of geometry data of a CAD system (e.g., AutoCAD) into that of another (e.g., CADdy) via e.g., STEP (Standard for the Exchange of Product Model Data) or the data exchange of business data (orders, shipping notifications, invoices, etc.) via UN/EDIFACT (United Nations Electronic Data Interchange for Administration, Commerce and Transport).

16 Peter Saint-Andre and Dave Smith, "XEP-0100: Gateway Interaction", accessed June 23, 2021, https:/xmpp.org/extensions/xep-0100.html.

17 "Have some quick conversations“, Quicksy, accessed June 23, 2021, https:/quicks y.im/.

18 "Be in the Zom", Zom, accessed June 23, 2021, https://zom.im/. 
Chapter 3. Interoperability obligation according to the EKEK

\subsection{Applicability of the EKEK to messenger services}

\subsubsection{Extension of the scope of application}

It has long been disputed whether messenger and other OTT communications services are subject to the traditional European legal framework for electronic communications. In Germany, the discussion was triggered by a ruling of the Cologne Administrative Court (VG Köln) ${ }^{19}$, which classified the webmail service Gmail as a telecomm5unications service within the meaning of $\$ 3$ No. 24 TKG (German Telecommunications Act of 22 June 2004). ${ }^{20}$ The question of whether this legal assessment is compatible with the European legal framework was referred to the ECJ. The ECJ ${ }^{21}$ rejected the functional understanding of the term "signal transmission" within the meaning of Art. 2(c) of the Framework Directive of 7 March $2002^{22}$ advocated by the Administrative Court of Cologne, and thus the application of the EU legal framework to Web mail services. From a strictly technical perspective, according to the ECJ, signal transmission is carried out exclusively by Internet access and communications network providers. It was not sufficient for this characteristic to be affirmed "that the provider of the Internet service takes active steps in the sending and receiving of messages, whether by assigning to the e-mail addresses the IP addresses of the corresponding terminal equipment or by breaking down the messages into data packets and feeding them into the open Internet or receiving them from the open Internet so that they can be forwarded to their recipients." In its European Electronic Communications Code of 11 December 2018 $(\text { EECC })^{23}$, the EU has reversed the trend and decided to base the definition of electronic communications services on a "more functional approach" to regulation. Such an understanding of the term could also cover services other than traditional services that enable communication. The background to this paradigm shift is the changes in user behaviour that have

19 Verwaltungsgericht Köln, Judgment of 11 November 2015, Az. 21 K450/15.

20 Telekommunikationsgesetz, Bundesgesetzblatt I 2004, 1190.

21 European Court of Justice, Judgment of 13 June 2019, Case C-193/18, ECLI:EU:C:2019:498.

22 Directive (EU) 2002/21, Official Journal of European Commission, L 108/33 of 24 April 2002.

23 Directive (EU) 2018/1972, Official Journal of European Commission, L 321/36 of 17 December 2018. 
been observed in recent years. The European Commission has not failed to notice that voice telephony, text messaging and e-mail transmission services are increasingly being replaced by online services with equivalent functionality, such as Internet telephony, messaging services and Webbased e-mail services. The central feature of the new definitional approach is the abandonment of the characteristic of signal transmission. In the future, electronic communications services are to include three types of services, some of which may overlap. According to Art. 2 No. 4 EECC an electronic communication service is:

"a service normally provided for remuneration via electronic communications networks, which encompasses, with the exception of services providing, or exercising editorial control over, content transmitted using electronic communications networks and services, the following types of services: (a) 'internet access service' as defined in point (2) of the second paragraph of Article 2 of Regulation (EU) 2015/2120; (b) interpersonal communications service; and (c) services consisting wholly or mainly in the conveyance of signals such as transmission services used for the provision of machine-to-machine services and for broadcasting".

\subsubsection{Messenger services as interpersonal communication services}

Messenger services can obviously only fall into category b). According to Art. 2 No. 5 EECC, an "interpersonal communication service" is

"a service normally provided for remuneration that enables direct interpersonal and interactive exchange of information via electronic communications networks between a finite number of persons, whereby the persons initiating or participating in the communication determine its recipient(s) and does not include services which enable interpersonal and interactive communication merely as a minor ancillary feature that is intrinsically linked to another service".

Recital 17 generally places messaging services in the category of interpersonal communications services. However, the most important messenger services available on the market must be examined to determine whether they meet the criteria of Article 2 No. 5 EECC.

With messenger services, users are firstly given the opportunity to reply. This means that communication is interactive. This feature distinguishes messenger services from linear services such as broadcasting, which ad- 
dresses its content to users as a one-to-many service. Secondly, no other person is involved in the exchange of information between these people. Communication is therefore direct and interpersonal. Third, the users themselves determine all the people involved in the communication process. For example, they must enter the telephone number as an identifier in order to reach the desired addressee.

Fourth, a finite number of people also participate in communication using messenger services. In the case of Telegram, there is the possibility to send messages to an unlimited number of users via the so-called "channels". However, this broadcasting function is only a partial function of a messenger service, whose main function remains the transmission of messages to a finite group of recipients. This is because the "sender" of the communication content determines all participants. WhatsApp, for example, has a maximum group size of 256 , which has been increased from 100. Fifth, the communication process takes place in messenger services via electronic communication networks. It is not decisive here that these are not networks of the messenger service providers, but of the Internet access service providers. ${ }^{24}$

Sixthly, it could still be questionable whether the characteristic of remuneration is present in the case of messenger services. This is because no direct monetary payments are made for these services. Rather, in the case of WhatsApp, Facebook Messenger and Skype, personal data is disclosed, or data is made available in return for the use of the services. According to recital 16 , however, this should be sufficient to be able to regularly assume that a payment has been made. The Telegram and iMessage services also meet the remuneration criterion. Telegram is financed by donations. iMessage is part of the system software, which is paid for in the purchase price. According to the provider, no personal data is processed or sold for either service.

Finally, as a seventh characteristic, Art. 2 No. 5 EECC requires that the service to be assessed is a communication service in its main function. As an example of a service that only enables a subordinate secondary function, Recital 17 mentions a communication channel in online games. The purpose of this characteristic is to prevent over-extension of the scope of the directive and regulation. In particular, content providers are not to be covered, as can be seen from Art. 2 No. 4 EECC. Whether this characteristic is present can only be decided in individual cases. Facebook Messenger, for example, was included in the user interface of the Facebook portal for a 
long time. Today, it is an independent service. The application can be downloaded via an app store. This should be sufficient to assign the service more than just a subordinate secondary function. With regard to the WhatsApp or Skype services, for example, there is no doubt from the outset that the requirements of Art. 2 No. 5 EECC are met.

Table: Characteristics of messenger services

\begin{tabular}{|c|c|c|c|c|c|c|c|}
\hline 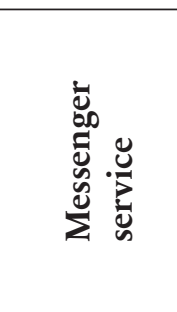 & 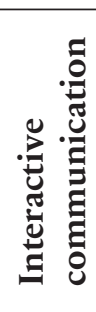 & 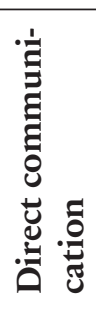 & 莺 & 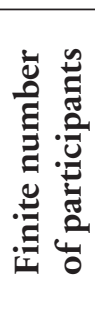 & 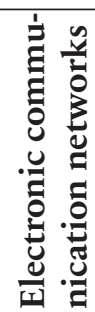 & 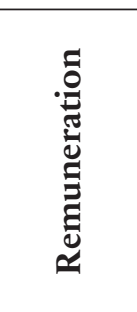 & 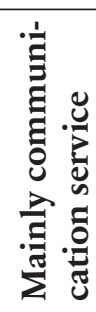 \\
\hline WhatsApp & Yes & Yes & Yes & Yes & Yes & Yes & Yes \\
\hline $\begin{array}{l}\text { Facebook- } \\
\text { Messenger }\end{array}$ & Yes & Yes & Yes & Yes & Yes & Yes & Yes \\
\hline Skype & Yes & Yes & Yes & Yes & Yes & Yes & Yes \\
\hline Threema & Yes & Yes & Yes & Yes & Yes & Yes & Yes \\
\hline Telegram & Yes & Yes & Yes & Yes & Yes & No & Yes \\
\hline iMessage & Yes & Yes & Yes & Yes & Yes & $\begin{array}{l}\text { System } \\
\text { software }\end{array}$ & Yes \\
\hline
\end{tabular}

\subsubsection{Types of interpersonal communication services}

According to the EECC, there are two subcategories of interpersonal communications services: number-based and number-independent services. Since their distinction is of paramount importance for the application of numerous provisions of the EECC, the messenger services to be found on the market shall be assigned to these two categories in advance.

The decisive factor for the distinction is whether the respective service is either a

"service which connects with publicly assigned numbering resources, namely, a number or numbers in national or international numbering plans, or which enables communication with a number or numbers in national or international numbering plans". 
If the answer to this question is in the affirmative, the service is a numberbased interpersonal communications service in accordance with Art. 2 No. 6 EECC If the answer is no, the service is a number-independent interpersonal communications service in accordance with Art. 2 No. 7 EECC.

For classification purposes, it should be noted that the mere use of a number as an identifier cannot be equated with the use of a number to establish a connection with publicly assigned numbers. Only when the number is used to connect to a publicly assigned number is it a number-based interpersonal communications service. It is part of the regulatory course set by the EECC to subject number-based services to stricter requirement because they use publicly allocated numbering resources and establish end-to-end connectivity to end users via the (number) mechanism.

With regard to the messenger services examined here, only Skype opens up the possibility of reaching another end user via the public number space for a (small) fee. In this case, Skype is to be classified as a numberbased interpersonal communications service. The other services examined here are to be qualified as number-independent interpersonal communications services. This also applies to Skype if the service is used in such a way that calls are only made between Skype users.

\subsection{Interoperability of services according to EECC}

\subsubsection{Authorization to promote and ensure interoperability}

Requirements for promoting and ensuring the interoperability of services can be found in Art. 61(1) EECC. The measures shall serve to achieve the objectives set out in Art. 3 EECC. The national regulatory authorities or the other competent authorities in the case of Art.3(2) subpara. 1(b) and (c) are responsible for ordering them.

Interpersonal communications services are explicitly addressed in Article 61(2) subpara. 1(b) and (c) EECC. Notwithstanding any access obligations for companies with significant market power (cf. Art. 68 EECC), the authorities may take the measures specified in subparagraph 1(b) of the provision for number-based interpersonal communications services and the measures specified in subparagraph 1 (c) for number-independent interpersonal communications services. The addition of "in particular" makes it clear that the measures listed are not exhaustive. It is already clear from the wording that the obligation is not part of asymmetrical regulation. 


\subsubsection{Interoperability of number-based communication services}

Pursuant to Art. 61(2) subpara. 1(b) EECC, the regulatory authorities may impose obligations on companies subject to a general licensee and controlling access to end users to make their services interoperable. These must be justified cases. In addition, the obligation can be ordered only to the extent necessary.

Which companies are subject to a general license can be seen from Art. 12 EECC (cf. Art. 15(1) EECC). The term "general permit" is misleading. The natural usage of the term suggests that it means the general permission of a certain activity. However, according to the legal definition in Art. 2 No. 22 EECC, it refers to "the legal framework" by which rights for the provision of electronic communications networks or services are guaranteed and in which sector-specific obligations are laid down. However, the introduction of a general license by Art. 3(2) of the Licensing Directive of 24 April 2002 eliminated the obligation, dating back to monopoly times, for companies to obtain an explicit permit or license from the regulatory authority before carrying out their activities and exercising their rights. They were to be bound only by the provisions of the regulatory framework. The European legislator hoped that this would strengthen the internal market. However, the member states were given the power to introduce a (declaratory) reporting obligation for companies subject to a general license. In this way, an overview of the players active in the market could be maintained. The EECC maintains this conception (cf. Art. 12(3) EECC, recitals $42 \mathrm{f}$.).

With regard to number-based interpersonal communications services, Art. 12(2) EECC clarifies that there must also be no authorization or license required for the providers of these services prior to commencing their activities. This is because the services "may only be made subject to general authorization." Special obligations may only be imposed with regard to the specifications mentioned in Art. 13(2) EECC and the rights of use mentioned in Arts 46 and 94 EECC.

However, there is no comparable regulation for number-independent interpersonal communications services and thus messenger services. This raises the question of whether their providers are subject to the regulations for general authorizations at all, such as a notification requirement. Recital 44 answers this question by stating that it is "not appropriate" to apply these regulations. This makes it particularly clear that the European legislator subjects number-independent interpersonal communications services to a lower level of regulation than is the case for number-based interpersonal communications services. They are to be subject to obligations only 
if this is justified by a public interest. The reason given for this is that number-independent services do not benefit from the "use of public numbering resources" and do not participate in the "publicly secured interoperable ecosystem". This justification is not convincing, as it no longer does justice to the current economic and social significance of this category of services in comparison to number-based services. Nevertheless, an analogous application of Art. 13(2) EECC to number-independent services is out of the question. This is because, as recital 44 shows, the legislator has seen the regulatory problem, so there is no regulatory gap.

It can thus be stated that providers of number-independent interpersonal communications services are not companies subject to a general license. This means that ensuring the interoperability of messenger services in accordance with Art. 61(2) subpara. 1(b) EECC is generally ruled out from the outset. An exception applies only to messenger services such as Skype, insofar as the service uses publicly assigned numbers.

\subsubsection{Interoperability of number-independent communication services}

\subsection{3.a). Regulatory approach}

This does not mean, however, that providers of number-independent messenger services cannot in any case be required to make their services interoperable. However, the hurdle for this is significantly higher than is the case for number-based services. According to Art. 61(2) subpara. 1(c) of the EECC, the prerequisites for this are that, in a justified case, end-to-end connectivity between end users is threatened due to a lack of interoperability between interpersonal communications services and that the addressee of the obligation has a significant coverage and user base. These prerequisites and the possible legal consequences of ensuring interoperability are further specified in terms of content in Art. 61 EECC.

In procedural terms, a two-step approach is envisaged. First, the Commission determines which threats to connectivity in the internal market exist. On this basis, it also clarifies whether and with what instruments action can be taken to counter these threats. In a next step, the regulatory authority is responsible for deciding whether to take action in view of the national circumstances. In doing so, they must also be able to take action on their own initiative in order to ensure that the policy objectives listed in Art. 3 EECC are observed (cf. Art. 61(6) EECC). T This procedural sequence alone shows that the regulatory authorities have to overcome high hurdles if they want to impose an interoperability obligation. In this re- 
gard, however, it is an exaggeration to speak of a "regulation without teeth", because the assessment of whether the relevant factual prerequisites are met can change dynamically depending on market conditions. ${ }^{25}$

\subsection{3.b). Threats to connectivity between end users}

The European legislator's restraint with regard to number-independent interpersonal communications services is also shown by the fact that an interoperability obligation under Art. 61(2) subpara. 1(c) EECC, unlike in the cases of lit. a and lit. b, can only be considered if an "appreciable" threat to a regulatory objective of Art. 3 EECC can be identified. A higher danger threshold is required. End-to-end connectivity between end users must already be "threatened" by a lack of interoperability between interpersonal communications services.

End-to-end connectivity between end users is ensured when there is the possibility of communication between the end users. In the English-language version of Art. 61(2) subpara. 1(c) EECC, this classic task of telecommunications is vividly described when it speaks of "end-to-end connectivity between end-users". This terminology is similar to the "end-to-end interconnection of services" formula used in Article 5 (1) (2) (a) of the Access Directive of March $2002^{26}$, which is replaced in the EECC by the phrase "end-to-end connectivity". However, end-to-end connectivity between end users requires that the systems and technologies used are interoperable. That is, they must be capable of working together and exchanging information with each other or making it available to the user as efficiently as possible. Achieving interoperability is therefore also one of the classic objectives of telecommunications law. The European legal framework therefore has a number of instruments, such as the specification of an interface for the end-to-end connection or the standardization of technical standards, to ensure that this objective is achieved. ${ }^{27}$

However, it is questionable when a threat to connectivity can be assumed. The literature is cautious in this regard. Certainly, it cannot simply be pointed out here that conventional voice telephony in PSTN mode

25 Stefan Bulowski, Regulierung von Internetkommunikationsdiensten. Zur Anwendbarkeit des Telekommunikationsrechts auf Voice over IP, Instant Messaging und E-MailDienste (Baden-Baden: Nomos, 2019).

26 Directive (EU) 2002/19., Official Journal of European Commission, L 108/7 of 24 April 2002.

27 Directive (EU) 2018/1972, Recital 148. 
provides the necessary connectivity between end users. After all, this is not an interpersonal communications service. However, as can be seen from the wording, the provision is concerned precisely with ensuring the interoperability of these services ("lack of interoperability between interpersonal communications services"). The reference to the possibility of multihoming, i.e., the frequently observed parallel use of several number-independent interpersonal communication services such as WhatsApp and Facebook Messenger, does not lead anywhere either. ${ }^{28}$ This is true even if a limit on the reasonableness of the available multihoming service is read into the law in the event of serious data protection concerns. This is because it remains the case that each of these services is proprietary in its own right and does not have an end-to-end connection with another of these services. Recital 149 therefore also comments exclusively on interpersonal communications services: With regard to this category of services, end-to-end connectivity is "currently" present because end-users use number-based interpersonal communications services. However, it could not be ruled out that "future technical developments or increased use of number-independent interpersonal communications services" would lead to a significant threat to connectivity between end users. This could result in significant market entry barriers and obstacles to further innovation.

The latest market analysis by the Federal Network Agency indicates that the frequency of use of interpersonal communications services in Germany has already shifted sharply in the direction of number-independent services. WhatsApp is the most-used service by a wide margin at $85.4 \%$. Facebook Messenger follows this with 4\% and Instagram with 3.3\%. Only then comes Skype with 1.3\%. This is an interpersonal communication service that can (also) be used on a number-dependent basis. These data indicate that the market development referred to in recital 149 has already occurred in Germany. However, this alone is not sufficient to justify an interoperability obligation.

Rather, if interoperability problems arise, the procedure provided for in Art. 61(2) subpara. 2(ii). EECC must be followed. In this case, the Commission is first required to have BEREC assess the situation at the Union and Member State level. On the basis of this report, the Commission must then decide whether regulatory intervention by the regulatory authority is necessary. However, according to Art. 61 (2) subpara. 2(ii). EECC, this can

28 Jürgen Kühling, "What to do with OTT? - Die Regulierung von Gmail, WhatsApp \& Co. de lege ferenda", in Regulierung - Wettbewerb - Innovation, ed. Torsten Körber and Jürgen Kühling (Baden-Baden: Nomos, 2017), 181. 
only be considered if end-to-end connectivity between end users throughout the Union or in at least three Member States is threatened to a significant extent. If such intervention is contemplated, the Commission should, as a next step, adopt implementing measures specifying the nature and scope of any regulatory measures. For this purpose, an examination procedure pursuant to Art. 118(4) of the EECC is to be carried out.

\subsection{3.c). Providers with significant coverage and user base}

The addressees of an interoperability obligation can only be providers of number-independent interpersonal communications services that have "significant coverage and user base". According to Recital 151, notable should mean that the geographical coverage and the number of end users ensure a "critical mass" with regard to the objective of end-to-end connectivity to be achieved. Accordingly, interoperability obligations should not apply as a rule if providers with a limited number of end users or limited geographic coverage can make "only a marginal contribution" to achieving this objective. The market data of the Federal Network Agency suggest that the requirements are met for Germany, at least for the WhatsApp service belonging to Facebook. ${ }^{29}$

However, the regulatory authorities are not to determine whether a provider has significant market power within the meaning of Art. 63 et seq. EECC. This is because the interoperability obligation is designed as a symmetrical regulatory measure, as are the other possible orders to be imposed under Art. 61 EECC. This is indicated by recital 157, which states that obligations to ensure connectivity and interoperability could be imposed "irrespective of the designation as an undertaking with significant market power". This is confirmed by the systematic position of Art. 61 in the EECC. The provision is located in the chapter on "Access and Interconnection" (Arts $61 \mathrm{f}$. EECC), but not in the chapter on "Access Obligations for Companies with Significant Market Power" (Arts 63 ff. EECC.).

\subsection{3.d). Scope of the obligation}

With regard to the legal consequences of an order issued by the regulatory authority, Art. 61(2), (5) sentence 1 EECC emphasizes the principle of pro-

29 Bundesnetzagentur, Nutzung von OTT-Kommunikationsdiensten, 16. 
portionality, which also applies elsewhere in European law. Interoperability obligations may only be imposed to the extent "necessary" to ensure end-to-end connectivity between end users (subparagraph 1(c)) or may not exceed the "extent necessary" for this purpose (subpara. 2(i)). The regulatory authority may also only intervene in "justified cases". The objective of proportionality is also explicitly mentioned in (Art. 61(5) p. 1 EECC).

There is also a requirement that regulatory measures must be "objective, transparent, and non-discriminatory". These are also general requirements of access regulation under telecommunications law. The application of these criteria is governed by the procedures set out in Articles 23, 32 and 33 of the EECC (Art. 61(5) sentence 1 EECC). As part of the notification procedure governed by these provisions, the Member States shall ensure that the European Commission and the national regulatory authorities are informed of the intended obligation and are given the opportunity to comment on it. ${ }^{30}$

The evaluation obligation of the regulatory authorities in Art. 61(5) sentence 2 EECC is also to be understood as an expression of the principle of proportionality. According to this, they must review the results of the obligation and condition within five years of the measure's enactment and whether its amendment or repeal would be appropriate in light of changing circumstances. The results of this review must be announced (Art. 61(5) sentence 3 EECC).

Art. 61(2)(i) EECC allows the regulatory authority to attach conditions to the interoperability obligation. The provider concerned may be required, in order to ensure the interoperability of interpersonal communications services, to publish "relevant information" itself or to authorize its use, modification and further dissemination by public authorities or other providers. In this way, guidance can be provided so that, as stated in Art. 61(1) sentence 2 EECC, small and medium-sized enterprises and operators with a limited geographical reach can benefit from the obligations imposed.

In addition, the provider concerned may be required to use and implement in practice standards and specifications listed in the directory referred to in Art. 39(1) EECC or other relevant European or international standards. According to Recital 148, Member States shall encourage the use of the published standards or specification for the provision of services, technical interfaces or network function as strictly necessary to ensure the interoperability of services. 


\section{Chapter 4. Conclusion}

The interoperability of messenger services would allow users of different service providers to exchange messages, photos, videos, and many other data formats across domains. The concept of a federated system, specifically the use of the XMPP protocol, was presented as one form of a technical design option. XMPP was chosen because it is already used by WhatsApp (in a modified form), which would simplify interoperability. However, other protocols, e.g., Matrix Protocol (which offers a bridge to XMPP), are also suitable for an interoperable design. This concept was analysed in terms of its impact on competition, innovativeness, data privacy, and usability. In summary, the following can be stated for these four points:

Competition is likely to benefit significantly from an interoperability obligation, as network effects are reduced. ${ }^{31}$ This can be advantageous for smaller existing messenger services as well as facilitate the market entry for new developments. The unique selling point is thus no longer the size of the user base, but the extensiveness of the functionalities.

Innovativeness should not be restricted by an interoperability obligation based on a federated approach. With this approach, companies remain free to use their own (XMPP-based) protocols. Thus, no functionalities are lost. However, each company must create a gateway that can also interact with other domains based on the standardized XMPP protocol to enable data exchange.

Since at least two messenger services (more than two in the case of group messages) are involved for cross-domain data exchange, personal metadata is usually generated twice. From a data privacy perspective, however, this does not pose a problem, as the General Data Protection Regulation has established a sufficient level of protection regarding the generated personal data.

Users do not seem to see any clear advantage in an interoperability obligation so far. Thus, no further benefits are expected to arise from a usability perspective, although sending messages to multiple messengers does mean an increase in functionality.

From the perspective of consumer convenience, the introduction of a federated system proves to be the most reasonable solution, as the competition is strengthened, and innovation and usability are not restricted. However, meta-information will always be shared with multiple providers, but this should not be a problem from a data privacy perspective.

31 Bundesnetzagentur, Nutzung von OTT-Kommunikationsdiensten. 
In legal terms, the European and German legislators have opted for the possibility of an interoperability obligation. The Federal Network Agency is responsible for issuing such an order. It can issue this order if connectivity between end users is threatened due to a lack of interoperability between interpersonal telecommunications services. Providers of numberindependent interpersonal telecommunication services that have a significant coverage and user base can be considered as addressees. For Germany, a study by the Federal Network Agency on OTT communication services in Germany suggests - as mentioned - that these conditions are met for the messenger services belonging to Facebook. However, the interoperability obligation can only be imposed if the European Commission has taken the necessary enforcement measures beforehand and a planned order by the Federal Network Agency is in line with this. In addition, the principle of proportionality must be applied in each individual case, so that the issues discussed (competition, innovativeness, data privacy, and usability) must once again be weighed against each other.

\section{Bibliography}

Arnold, René, and Anna Schneider. "An App for Every Step: A psychological perspective on interoperability of Mobile Messenger Apps." 28th European Regional Conference of the International Telecommunications Society (ITS) (July/August 2017).

Arnold, R., A. Schneider and J. Lennartz. "Interoperability of interpersonal communications services - A consumer perspective.” Telecommunications Policy 44, no. 3 (April 2020). https://doi.org/10.1016/j.telpol.2020.101927.

Bitkom. "Zwei von drei Internetnutzern verwenden Messenger." Accessed June 23, 2021. https:/www.bitkom.org/Presse/Presseinformation/Zwei-von-drei-Internet nutzern-verwenden-Messenger.html.

Bulowski, Stefan. Regulierung von Internetkommunikationsdiensten, Zur Anwendbarkeit des Telekommunikationsrechts auf Voice over IP, Instant Messaging und E-Mail-Dienste. Baden-Baden: Nomos, 2019.

Bundesnetzagentur für Elektrizität, Gas, Telekommunikation, Post und Eisenbahnen. Nutzung von OTT-Kommunikationsdiensten in Deutschland. Bonn: Bundesnetzagentur für Elektrizität, Gas, Telekommunikation, Post und Eisenbahnen, 2020. https:/www.bundesnetzagentur.de/SharedDocs/Mediathek/Berichte/ 2020/OTT.pdf?_blob=publicationFile. 
Digitale Gesellschaft e.V. "Stellungnahme der Digitalen Gesellschaft e. V. zur Konsultation des Bundesministeriums der Justiz und für Verbraucherschutz zu Interoperabilität und Datenportabilität bei sozialen Netzwerken." Accessed June 23, 2021. https://digitalegesellschaft.de/2019/05/stellungnahme-der-digitalen-ges ellschaft-e-v-zur-konsultation-des-bundesministeriums-der-justiz-und-fuer-verbra ucherschutz-zu-interoperabilitaet-und-datenportabilitaet-bei-sozialen-netzwerke $\mathrm{n} /$.

Katz, Michael L., Carl Shapiro. "Systems Competition and Network Effects." Journal of Economic Perspectives 8, no. 2 (Spring 1994): 93-115. https://doi.org/10.1257 /jep.8.2.93.

Kühling, Jürgen. "What to do with OTT? - Die Regulierung von Gmail, WhatsApp \& Co. de lege ferenda." In Regulierung - Wettbewerb - Innovation, edited by Torsten Körber and Jürgen Kühling, 165-185. Baden-Baden: Nomos, 2017.

Quicksy. „Have some quick conversations.“ Accessed June 23, 2021. https://quicksy. $\mathrm{im} /$.

Saint-Andre, Peter and Dave Smith, "XEP-0100: Gateway Interaction." Accessed June 23, 2021. https://xmpp.org/extensions/xep-0100.html.

Wegner, Peter. "Interoperability." ACM Computing Surveys 28, no. 1 (March 1996). 285-287. https://doi.org/10.1145/234313.234424.

Zom. „Be in the Zom.“ Accessed June 23, 2021, https://zom.im/. 
